\title{
PSEUDO-ISOTOPIES OF ARCS AND KNOTS
}

\author{
CARL D. SIKKEMA ${ }^{1}$
}

\begin{abstract}
The purpose of this paper is to show that if an arc or simple closed curve contains a nontrivial Wilder arc, then it is not possible to transform a straight line segment onto the arc or a knot onto the simple closed curve. The proof uses the fact that every knot has a unique finite decomposition into prime knots.
\end{abstract}

A pseudo-isotopy is a homotopy $F_{t}, 0 \leqq t \leqq 1$, such that $F_{t}$ is a homeomorphism for $t<1$. An arc $A$ is locally unknotted [4] if for each $x \in A$ there is a neighborhood of $x$ in $A$ that lies on a disk. It is known [5], [6], [7] that if $M$ is a 2-manifold, a locally unknotted arc or a locally unknotted simple closed curve in $E^{3}$ and if $\varepsilon>0$, then there is a polyhedral manifold $N \subset E^{3}$, homeomorphic to $M$, and an $\varepsilon$-pseudo-isotopy $F_{t}$ of $E^{3}$ that transforms $N$ onto $M$. In addition, $F_{0}=1, F_{1} \mid N$ is a homeomorphism of $N$ onto $M$ and the set of points whose preimages under $F_{1}$ are nondegenerate is a zero-dimensional subset of the set of wild points of $M$.

An arc is mildly wild if it is the union of two tame arcs. A Wilder arc [3] is a mildly wild locally peripherally unknotted [4] arc. See Example 1.4 of [1]. The following theorems show that the result above cannot be extended to all arcs and simple closed curves.

THEOREM 1. If $A$ is an arc that contains a nontrivial Wilder arc, $I$ is a straight line segment, and $F_{t}: E^{3} \rightarrow E^{3}$ is a pseudo-isotopy, then $F_{1}(I) \neq A$.

THEOREM 2. If $S$ is a simple closed curve that contains a nontrivial Wilder arc, $k$ is a knot, and $F_{t}: E^{3} \rightarrow E^{3}$ is a pseudo-isotopy, then $F_{1}(k) \neq S$.

However, it is trivial that any arc or simple closed curve can be transformed by a pseudo-isotopy onto a straight line segment or a circle, respectively.

Theorem 1 follows immediately from Theorem 2 .

Proof of Theorem 2. Let $C$ be a geometric cone in $E^{3}$ with vertex $p$ and square base $F_{0}$. For each integer $i>0$, let $F_{i}$ be the intersection of $C$ and the plane parallel to $F_{0}$ that is half way between $F_{i-1}$ and $p$. Let $C_{i}$ be

\footnotetext{
Received by the editors February 11, 1971.

AMS 1970 subject classifications. Primary 55A25, 55A30; Secondary 57A10.

Key words and phrases. Arc, knot, pseudo-isotopy, Wilder arc, simple closed curve.

${ }^{1}$ This research was partially supported by the National Science Foundation, Grant GP-11943.
} 
the closed subset of $C$ between $F_{i}$ and $F_{i+1}$. Since $S$ contains a nontrivial Wilder arc, we can assume without loss of generality that $S \cap C_{0}$ is a straight line segment $A_{0}$ and, for $i>0$, that $S \cap C_{i}$ is a polyhedral arc $A_{i}$ that meets the boundary of $C_{i}$ only at the endpoints of $A_{i}$ and the midpoints of $F_{i}$ and $F_{i+1}$ such that $A_{i}$ plus an arc on the boundary of $C_{i}$ is a nontrivial knot.

Suppose there is a knot $k$ in $E^{3}$ and a pseudo-isotopy $F_{t}: E^{3} \rightarrow E^{3}$ such that $F_{1}(k)=S$. For each integer $i \geqq 0$, let $N_{i}$ be a regular neighborhood of $A_{i}$ in $C_{i}$ such that $N_{i} \cap F_{i+1}=N_{i+1} \cap F_{i+1}$.

Let $n$ be a positive integer. There exists a $t<1$ such that

$$
F_{t}(k) \cap\left(\bigcup_{0}^{n+1} C_{i}\right) \subset \bigcup_{0}^{n+1} N_{i} .
$$

We may suppose without loss of generality that $F_{t}(k) \cap C_{0}=A_{0}$. Then there is a homeomorphism $h: E^{3} \rightarrow E^{3}$ such that

$$
h\left(A_{0}\right)=\bigcup_{0}^{n} A_{i}=h\left(F_{t}(k)\right) \cap\left(\bigcup_{0}^{n} C_{i}\right) .
$$

Since $k$ and $h F_{t}(k)$ are equivalent, $k$ can be decomposed into at least $n$ knots for every $n$. But every knot has a unique finite decomposition into prime knots [2]. Hence no such pseudo-isotopy exists.

\section{REFERENCES}

1. R. H. Fox and E. Artin, Some wild cells and spheres in three-dimensional space, Ann. of Math. (2) 49 (1948), 979-990. MR 10, 317.

2. R. H. Fox, A quick trip through knot theory, Topology of 3-Manifolds and Related Topics (Proc. Univ. of Georgia Inst., 1961), Prentice-Hall, Englewood Cliffs, N.J., 1962, pp. 120-167. MR 25 \#3522.

3. R. H. Fox and O. G. Harrold, The Wilder arcs, Topology of 3-Manifolds and Related Topics (Proc. Univ. of Georgia Inst., 1961), Prentice-Hall, Englewood Cliffs, N.J., 1962, pp. 184-187. MR 25 \#3519.

4. O. G. Harrold, Jr., Combinatorial structures, local unknottedness, and local peripheral unknottedness, Topology of 3-Manifolds and Related Topics (Proc. Univ. of Georgia Inst., 1961), Prentice-Hall, Englewood Cliffs, N.J., 1962, pp. 71-83. MR 28 \#1597.

5. L. V. Keldyš, Topological imbeddings and pseudo-isotopy, Dokl. Akad. Nauk SSSR 169 (1966), 1262-1265=Soviet Math. Dokl. 7 (1966), 1078-1082. MR 34 \#3556.

6. - Topological imbeddings in a manifold and pseudoisotopy, Mat. Sb. 71 (113) (1966), 433-453; English transl., Amer. Math. Soc. Transl. (2) 84 (1969), 253-275. MR 34 \#6784.

7. - Topological imbeddings of simple arcs and closed curves in $E^{3}$, Dokl. Akad. Nauk SSSR 185 (1969), 513-516=Soviet Math. Dokl. 10 (1969), 376-379. MR 39 \#2141.

Florida State University, Tallahassee, Florida 32306 